\title{
RETRACTED ARTICLE: Ultrasonic piezonuclear reactions in steel and sintered Ferrite bars
}

\author{
F. Cardone $\cdot$ A. Carpinteri $\cdot$ A. Manuello $\cdot$ \\ R. Mignani - A. Petrucci - E. Santoro • \\ M. Sepielli
}

Received: 30 October 2012/ Accepted: 7 March 2014/Published online: 4 June 2014

(C) The Author(s) 2014

This article has been withdrawn by the Publisher and the Society in agreement with the Editor-in-Chief due to conflict of interest reasons. In a commitment to scientific integrity we decided to withdraw the article as the editorial process had been compromised.

F. Cardone

Istituto per lo Studio dei Materiali Nanostrutturati (ISMN-

CNR), Via dei Taurini, 00185 Rome, Italy

F. Cardone - R. Mignani

Istituto Nazionale di Alta Matematica F. Severi Città

Univ., P.le Aldo Moro 2, 00185 Rome, Italy

F. Cardone $\cdot$ R. Mignani - A. Petrucci $(\bowtie)$

Dipartimento di Fisica E.Amaldi, Università degli Studi

Roma Tre, Via della Vasca Navale, 84, 00146 Rome, Italy

e-mail: petrucciandr@gmail.com

A. Carpinteri · A. Manuello

Dipartimento di Ingegneria Strutturale, Edile e

Geotecnica, Politecnico di Torino, Corso Duca degli

Abruzzi, 24, 10129 Turin, Italy

E. Santoro - M. Sepielli

Unità Tecnica Tecnologie e Impianti per la Fissione e la Gestione del Materiale Nucleare (UTFISST) - ENEA, Via Anguillarese, 301, Santa Maria di Galeria, 00123 Rome, Italy 